\title{
AMENDMENTS
}

\section{Author Correction: Ancient admixture from an extinct ape lineage into bonobos}

Martin Kuhlwilm (D), Sojung Han (1), Vitor C. Sousa, Laurent Excoffier (1) and Tomas Marques-Bonet (1)

Correction to: Nature Ecology \& Evolution https://doi.org/10.1038/s41559-019-0881-7, published online 29 April 2019.

In the version of this article originally published, a funding acknowledgement was missing for Tomas Maques-Bonet. The original funding statement was: “T.M.-B. was supported by MINECO BFU2014-55090-P (FEDER), a U01 MH106874 grant, the Howard Hughes International Early Career programme, Obra Social 'La Caixa' and Secretaria d'Universitats i Recerca del Departament d'Economia i Coneixement de la Generalitat de Catalunya." It has been updated to: “T.M.-B. was supported by BFU2017-86471-P (MINECO/ FEDER, UE), a U01 MH106874 grant, the Howard Hughes International Early Career programme, Obra Social 'La Caixa' and Secretaria d'Universitats i Recerca and CERCA Programme del Departament d'Economia i Coneixement de la Generalitat de Catalunya (GRC 2017 SGR 880)." The error has been corrected in the HTML and PDF versions of this article.

Published online: 14 May 2019

https://doi.org/10.1038/s41559-019-0919-x 